\title{
Traumatic intrusion of primary teeth and developmental defects in successor teeth
}

\author{
Abstracted from \\ Altun C, Cehreli ZC, Güven G, Acikel C. \\ Traumatic intrusion of primary teeth and its effects on the permanent successors: \\ a clinical follow-up study. Oral Surg Oral Med Oral Pathol Oral Radiol Endod 2009; 107: 493-498 \\ Address for correspondence: Ceyhan Altun, Department of Paediatric Dentistry, \\ Centre for Dental Sciences, Gulhane Medical Academy, Etlik/ Ankara, Turkey. E-mail: ceyhanaltun@yahoo.com
}

\section{Question: What are the most likely effects of traumatic intrusion of primary teeth on the permanent successor teeth?}

Design This was a clinical observational study of primary incisor trauma, in children of under 4 years of age at the time of injury, with a 7-year follow up period.

Cohort selection Children treated for intrusive injuries (as defined by World Health Organization guidelines) of primary incisors in a paediatric clinic.

Exposure measurement Patient's age, sex, cause of trauma, site and number of primary teeth involved, and nature of immediate treatment were recorded. Photographs and radiographs were taken. At 6-12month intervals clinical and radiographical examinations recorded the process of re-eruption, and any post-traumatic changes to the intruded primary teeth: namely, pulpal necrosis, root resorption, ankylosis, or obliteration of the pulp canal. Developmental defects were recorded on the fully erupted successor teeth, namely enamel hypoplasia, malformation of tooth and/ or root, and site of eruption.

Data analysis Chi-squared tests were used to determine significant differences between the patient's age at diagnosis; subsequent changes to the intruded primary teeth; and any developmental defects in the successor teeth.

Results Complete data were obtained over a 7-year followup period, for 78 children (138 teeth) who were aged between 12 and 48 months at initial examination. Thirty-six of these teeth, in 23 children, were extracted at the first visit because of extensive lateral luxation as well as intrusion. Of the remaining (unextracted at initial visit) 102 intruded primary incisors, $78 \%$ fully re-erupted, $15 \%$ partially re-erupted, and only $7 \%$ remained impacted. Post-traumatic consequences were recorded in $54 \%$ of the teeth. Over half of the permanent successors (74 out of the initial sample of 138 traumatised primary incisors) were found to have one or more developmental disturbances: enamel hypoplasia (28.3\% of all incisors); dilacerations ( $16.7 \%$ of all incisors); or ectopic eruption (16.7\% of all incisors).

Conclusions The study did not find any correlation between the child's age at the time of the intrusion injury and the frequency of subsequent developmental disturbances.

\section{Commentary}

It is likely that clinicians who provide dental care for children will have to manage traumatic intrusion of primary incisors at some time. Overall trauma to primary incisors is common (seen in around $30 \%$ of children by the age of 7 years ${ }^{1}$ ). However, although intrusion injuries are uncommon in adults, it is likely that around one in five of injuries to primary teeth will be intrusive (17\% in one hospital based study ${ }^{2}$ ). There is still debate around how best to manage these teeth.

This 7-year clinical followup of such injuries presents information on the consequences for the intruded primary teeth, and also on what is recognised as one of the more distressing sequelae of this injury; the effect on the permanent successors, whose follicles lie slightly above and palatal to the root apex of the primary incisors.

Seventy eight children with 138 traumatised primary incisors fulfilled the inclusion criteria. Thirty six of these teeth, in 23 children, were extracted at the first visit, due to extensive lateral luxation as well as intrusion. In keeping with other reports, this study found that the majority of intruded primary incisors, where immediate extraction was not indicated, re-erupted when simply observed (78\% of the remaining 102). However, 54\% developed post traumatic consequences, with the most common being pulpal necrosis in $31 \%$ of the 102 teeth (managed either with pulpectomy or extraction). Over half of the permanent successors (74 out of the initial sample of 138 traumatised primary incisors) were found to have one or more developmental disturbances: enamel hypoplasia $(28.3 \%$ of all incisors); dilacerations (16.7\% of all incisors); ectopic eruption (16.7\% of all incisors).

But does this actually help to inform as to how best to manage the intruded primary incisor? Can they always be left and observed, unless excessively laterally luxated? Recent guidance ${ }^{3}$ states that if radiographic examination indicates (by way of the intruded tooth appearing lengthened relative to its antimere) that the apex of the intruded incisor has been pushed palatally into the follicle of the succedaneous permanent incisor, then the primary incisor should be extracted, to minimise further damage to the tooth germ. However, there is little evidence to support this and there are the associated risks of causing further distress to the child through the extraction and iatrogenic damage to the underlying permanent tooth. In this study, it is stated that radiographs were taken of all children at the initial visit. It would be of great use to clinicians to know if there was a positive association between palatal displacement of the intruded primary incisor, and the $54 \%$ of permanent successors which subsequently 


\section{PAEDIATRIC DENTISTRY}

showed signs of developmental disturbance. This could then inform a further study to determine if immediate extraction of these teeth reduces the prevalence of developmental defects in the permanent incisors.

Nicola PT Innes

Preventive and Children's Dentistry Section, Dundee Dental Hospital and School, University of Dundee, Dundee, Scotland, UK

1. Andreasen JO, Ravn JJ. Epidemiology of traumatic dental injuries to primary and permanent teeth in a Danish population sample. Int / Oral Surg 1972; 1: 235-237. Borum MK, Andreasen IO Sequelae of trauma to primary maxillary incisors. I. Complications in the primary dentition. Endod Dent Traumatol 1998; 14: 31-44.

. Flores MT, Malmgren B, Andersson L, et al. Guidelines for the management of

3. Flores MT, Malmgren B, Andersson L, et al. Guidelines for the management of
traumatic dental injuries. III. Primary teeth. Dent Traumatol 2007; 23: 196-202.

\section{Practice points}

- The majority of intruded primary incisors where immediate extraction is not indicated will re-erupt if simply observed.

- Following the intrusion of a primary incisor, just over half of permanent successors incisors are likely to have one or more developmental defects.

- There is a need for further evidence as to whether extraction of intruded primary incisors where the apex impinges on the follicle of the permanent successional incisors, reduces the frequency of developmental defects in that tooth, and to a degree which would justify the additional trauma of the extraction to the child.

Evidence-Based Dentistry (2009) 10, 70-71. doi:10.1038/sj.ebd.6 400663 